\title{
Spatiotemporal Topic Association Detection on Tweets
}

\author{
Zhi Liu, Yan Huang \\ Department of Computer \\ Science and Engineering \\ University of North Texas, \\ Denton, TX, 76201 \\ zhiliu@my.unt.edu \\ yan.huang@unt.edu
}

\author{
Joshua R. Trampier \\ Department of Defense \\ Springfield, VA 22150, USA \\ Joshua.R.Trampier@nga.mil
}

\begin{abstract}
The analysis of Twitter data can help to predict or explain many real world phenomena. The relationships among events in the real world can be reflected among the topics on social media. In this paper, we propose the concept of topic association and the associated mining algorithms. Topics with close temporal and spatial relationship may have direc$\mathrm{t}$ or potential association in the real world. Our goal is to mine such topic associations and show their relationships in different time-region frames. We propose to use the concepts of participation ratio and participation index to measure the closeness among topics and propose a spatiotemporal index to calculate them efficiently. With the topic filtering and the topic combination, we further optimize the mining process and the mining results. The algorithms are evaluated on a Twitter dataset with 27,956,257 tweets.
\end{abstract}

\section{Categories and Subject Descriptors}

H.2.8 [Database Management]: Database ApplicationsData Mining

\section{Keywords}

Hashtag, Participation Index, Topic Association

\section{INTRODUCTION}

The analysis of Twitter data can help to predict or explain phenomena in many real world applications [1, 3]. Topics on Twitter may potentially be associated in space and time. For example, the hashtags related to the Independence Day \#july4th are closely related to the hashtags \# firework and \#beer in the beginning of July, which directly reveals how people celebrate the Independence Day. The detection of such topic associations can help to improve on-line services such as news recommendation and explain the relationship among real world events.

The goal of this paper is to detect the associations between hashtags (topics) by analyzing the time and location

Permission to make digital or hard copies of all or part of this work for personal or classroom use is granted without fee provided that copies are not made or distributed for profit or commercial advantage and that copies bear this notice and the full citation on the first page. Copyrights for components of this work owned by others than ACM must be honored. Abstracting with credit is permitted. To copy otherwise, or republish, to post on servers or to redistribute to lists, requires prior specific permission and/or a fee. Request permissions from permissions@ acm.org.

SIGSPATIAL'16, October 31-November 03, 2016, Burlingame, CA, USA

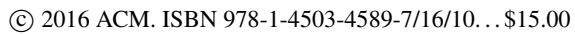

DOI: http://dx.doi.org/10.1145/2996913.2996933 distributions of tweets which contain these hashtags. We define the topic set of each tweet as the hashtags it contains. So each topic can appear in a number of tweets and each tweet can contain several topics. For example, the right side of Figure 1 shows two tweets. The first tweet is related to the topic/hashtag \#Amazon and the second one contains two topics \#Job and \#Houston. The left side of Figure 1 shows three groups of hashtags represented by three colors. The weights in the graph measure the closeness between the hashtags and the calculation of these weights will be discussed in section 3. Using these relationships, when a user talks about certain topics, such as \#job, we can recommend the tweets from other related topics based on the topic associations (e.g. $\{\# j o b, \#$ hiring, \#tweetmyjobs\}) so that the users can get more comprehensive information of what they want. Such close correlated topic associations can also reflect the relations among real world events. For example, the mining process reveals a topic association of $\{\#$ nbafinals, \#cle, \#gsw\}. As a matter of fact, hashtags \#cle and \#gsw are the two teams in the NBA finals 2015. The mining process reveals these three topics appearing in space and time proximity.

However, the detection of topic associations has several challenges. First, we need an appropriate measurement to evaluate the closeness between two or more topics. The second challenge is that each topic may have a large number of related tweets. We need algorithms scalable to large number of tweets and topics. More importantly, the closeness of topic associations can be different in different time and region. So we must take time-region frame into consideration to provide a more accurate measurement, which can make the calculation more complex and expensive. Finally, the detection may result in a large number of topic association$\mathrm{s}$. So it is necessary to filter out the less interesting topic associations.

Topic Association First we define the closeness among topics by examining the co-occurrence of tweets containing these topics. Closely related topics may tend to be talked in the similar time and region. Based on this idea, we define the participation index of topic associations. If the tweets of a set of topics tend to be found in the same time and region, they will have a higher participation index. We evaluate this concept in our experiments by comparing the measuring results of participation index with the human judgment. Based on the concept of participation index, we propose three different types of queries to help users to extract topic associations with different semantics in different time-region frames. 


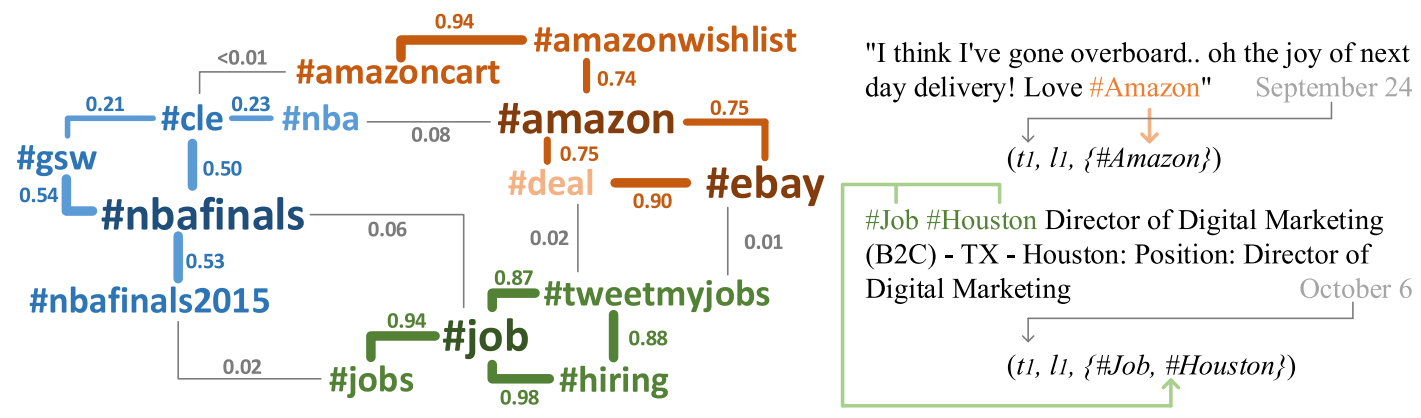

Figure 1: Closeness of topics and topic related tweets.

Mining Topic Association One of the challenges is that the number of tweets can be very large as well as the topics and their combinations. For example, in the sample we collected, there are 364,333 geo-tagged tweets that contain the hashtag \#job in the U.S. in July, 2015. So how to develop an efficient algorithm to help to calculate the participation index of a set of topics will be the most important step. Here we propose a multi-layer geographic index and a time index to help us to filter the topic sets under the threshold quickly to calculate the participation index.

Optimization Another challenge in our work is the number of topics. Our dataset contains 492,492 hashtags. Any subset of these hashtags can be a potential topic association. To deal with the exponential nature of the hashtag associations, we propose two methods to optimize the the mining algorithm: topic filtering and topic combination. In the topic filtering, we will remove some topics which are not affected by other topics or real world events. These topics participate in a large number of associations and the resulting associations are not interesting. Furthermore, we propose an algorithm to combine similar topics to further reduce the complexity.

This paper makes the following contributions:

- We define the participation index of a set of topics by measuring the time and location distributions of tweets containing the topics; We define three types of queries to answer questions related to topic associations;

- By introducing the time and location indexes, we propose an efficient algorithm to calculate the participation index;

- Topic filtering and topic combination methods are developed to optimize the querying results;

- We test our algorithm on a large Twitter dataset which contains 27,956,257 tweets and 492,492 hashtags. The results demonstrate that our method is effective and efficient.

The rest of the paper is organized as follows: In section 2 we introduce the related work. Section 3 provides the definitions of relationship between hashtags and three types of queries. In section 4 , we describe the mining algorithm and in section 5 , we propose two optimization methods. We evaluate our algorithm in section 6 .

\section{RELATED WORK}

In the recent years, many researchers have shown interest in topic, trend, events and their correlation on social media. Different methods have been used to analyze tweet content including machine learning algorithms, language model, feature-based algorithms, information retrieval and many more with help from spatiotemporal data mining. Petrovic et al. [15] employ a locality-sensitive hashing (LSH) to detect first story from a stream of tweets. The proposed approach uses hash table to organize new similar tweets in an existing story or labels. Although LSH has been used in nearest neighbor search applications, Protrovic's work makes event detection possible on large-scale tweets dataset. This approach does not differentiate the nature of events and content such as local event, disaster or news. Sakaki et al. [16, 17] classify tweets based on features such as keywords or word number and use a probabilistic spatio-temporal model to detect earthquakes from tweet content. The event location is estimated using Kalman filtering and particle filtering. The assumption of Sakaki's approach, is that only one event takes place in one region at a time and users know the event to set up keyword queries in advance.

Budak et al. [5] proposed a location-topic pair index using both tweet location and user location to detect geo-trends, or trends in different geographic locations in a sliding window. Kamath et al [10] did a thorough study of a tweet's hashtags as a representation of its topic. They did an analysis of the global footprint of hashtags and an exploration of the spatial constraints on hashtag adoption. They measure the hashtag's spatial propagation properties: focus, entropy, and spread. Lee et al. [12] tried to detect events such as traffic jams by using geographical coordinates of geotagged tweets and monitoring the geographical pattern of tweets. Sugitani et al. [20] use a different approach to detect events by using spatio-temporal clustering techniques. Schulz et al. [18] are the first in using a multi-indicator approach to get accurate geographic location of tweets and Twitter users.

Analysis of the time, location and hashtags of tweets can help to extract more meaningful information from real world events, trends, and roles in social networks. In [4], the authors detect trends which related to locations. Hashtags are also defined as topics of tweets here as well as [21] and three different thresholds are introduced to define the correlation between topics and locations. In [22], the authors propose a linear regression based approach to predict the spread of an idea in a given time frame. The hashtags are taken as the idea of tweets. Yang et al. [24] propose measurements to analyze the main factors of how users select hashtags and a machine learning model is used to help to predict the future adoption of hashtags of users. Kywe et al. [11] study the time distribution of hashtags, and then the authors propose a hashtag recommendation method based on collaborative filtering. In [13], the authors propose a geovisual analytics approach to leveraging Twitter in support of crisis management. The hashtags which are related to locations are used to extract more location information of tweets. By study- 


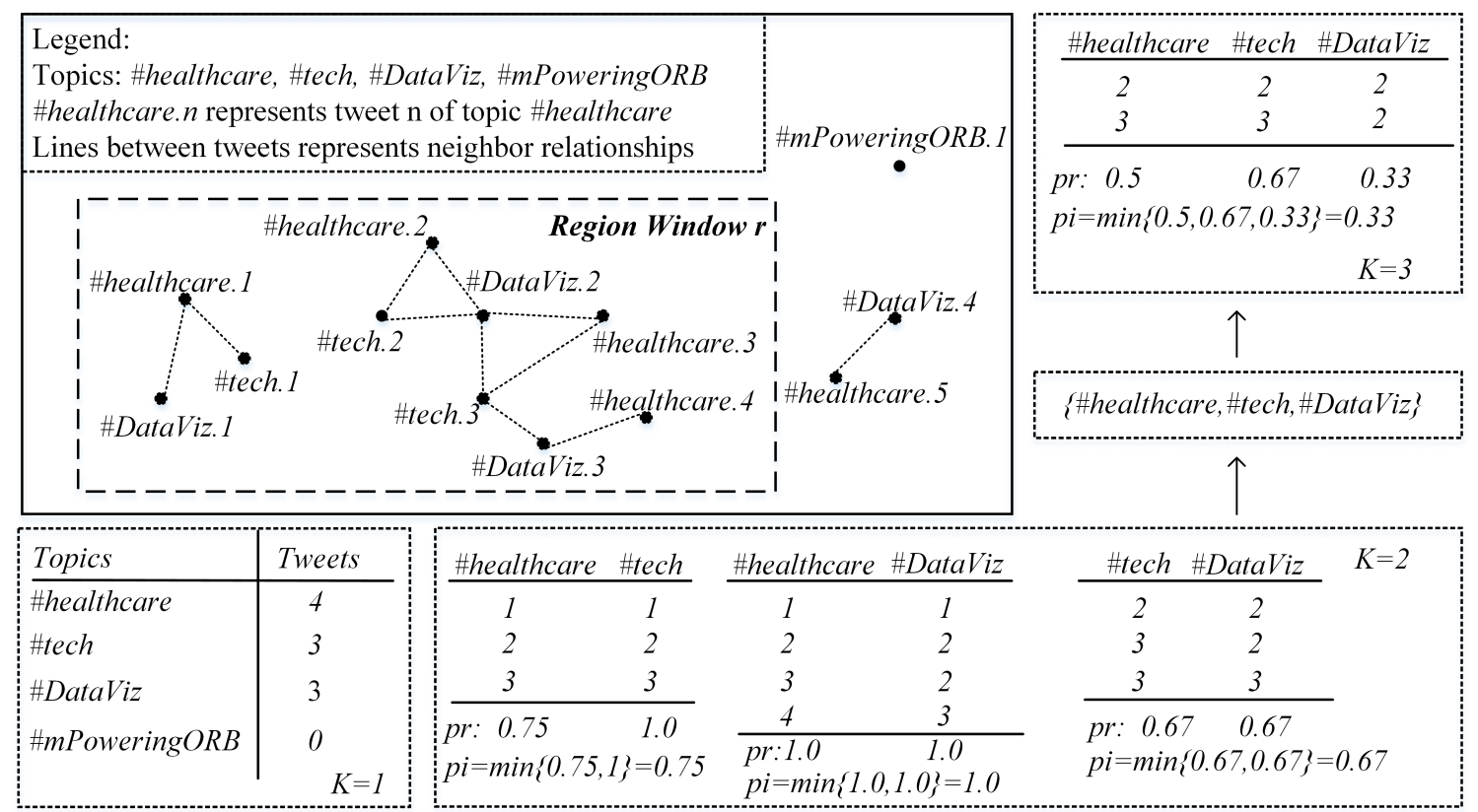

Figure 2: Mining topic association in time-region frame $\langle w, r\rangle$. In this case, we set the time window $w$ as the whole time line, which means every tweet is in the time window, and then we set $r$ as the area circled in the dashed line. There are four hashtags (topics) in this example: \#healthcare, \#tech, \#DataViz, and \#mPoweringORB. Each topic is contained in several tweets. We firstly generate 3 size -2 (each topic association contains two topics) topic association and then generate a size -3 topic association.

ing the tweets related to 2011 London riots, Glasgow et al. [8] analyze emergent social networks directly relating to response to crisis. Their study shows that the hashtag lifespan may relate to social behaviors and social networks coupled to crisis response. Carter et al. [6] propose a method for translating hashtags, which builds on methods from information retrieval.

Co-location patterns $[9,25,14]$ and efficient algorithms have been studied by various researchers. An initial summary of results on general spatial co-location mining was proposed in [19]. The authors proposed the notion of userspecified neighborhoods in place of transactions to specify group of spatial items. By doing so, they can adopt traditional association rule mining algorithms, i.e., Apriori algorithm [2] to find spatial co-location rules. An extended version of their approaches was presented in [9].

\section{PROBLEM DEFINITION}

In this section, we introduce the definition of participation index of topic association and three kinds of topic association queries. Table 1 gives the notations used in this paper.

Table 1: Notations and parameters

\begin{tabular}{|c|c|}
\hline$v_{i}$ & Tweet $i$ \\
\hline$t_{i}, l_{i}, \mathcal{H}_{i}$ & The time, region and hashtags of tweet $v_{i}$ \\
\hline$w, r$ & Time and region windows \\
\hline$W, R$ & Set of time and region windows \\
\hline$h, \mathcal{H}$ & Topic $h$ and a set of topics $\mathcal{H}$ \\
\hline$t, d, \theta, k$ & Thresholds in query \\
\hline
\end{tabular}

A tweet $v_{i}$ is represented by a triple $\left(t_{i}, l_{i}, \mathcal{H}_{i}\right)$, where $t_{i}$ is the time when it is shared, $l_{i}$ is the location where it is shared, and $\mathcal{H}_{i}$ is a set of hashtags contained in the tweet. For example, in the Figure 1, the triple for the first tweet $v_{1}:\left(t_{1}, l_{1}, \mathcal{H}_{1}\right)$ has the time information September 24 , location information $l_{1}$, and the topics list $\{$ \#amazon $\}$.

Definition[Topic] The topics of each tweet are defined as the hashtags it contains. One tweet can relate to several topics.

Let $w$ and $r$ denote time and region windows, and we define the pair of $\langle w, r\rangle$ as a time-region frame. Each frame corresponds to a set of tweets $V_{\langle w, r\rangle}=\left\{v_{i} \mid t_{i} \in w \wedge l_{i} \in r\right\}$.

Definition[Related Tweets] For each topic, its related tweets are the tweets which contain this topic. So the set of related tweets of topic $h$ in a time-region frame $\langle w, r\rangle$ can be denoted as: $V_{\langle w, r\rangle}(h)=\left\{v_{i} \mid h \in \mathcal{H}_{i} \wedge t_{i} \in w \wedge l_{i} \in r\right\}$.

Definition[Topic Set] A topic set is a set of hashtags and denoted as: $\mathcal{H}=\left\{h_{1}, h_{2}, \ldots\right\}$.

\subsection{Calculating Participation Index}

Definition[Neighbor Relationship] Two tweets, $v_{i}$ and $v_{j}$, are neighbors when: 1) $\left|t_{i}-t_{j}\right| \leqslant \varphi$ and $\left|l_{i}-l_{j}\right| \leqslant \delta$ where $\varphi$ and $\delta$ are two user given thresholds on time and distance.

Definition[Supporting Tweet] A set $V=\left\{v_{1}, v_{2}, \ldots, v_{k}\right\}$ of $k$ tweets supports a size- $k$ topic set $\mathcal{H}=\left\{h_{1}, h_{2}, \ldots, h_{k}\right\}$ if tweets in $V$ are pairwise neighbors and $h_{i} \in \mathcal{H}_{i}$ for $i=$ $1,2, \ldots, k$. Each tweet $v_{i}$ in the set $V$ is called supporting $\mathcal{H}$ with $h_{i}$ and the predicate $s\left(v_{i}, h_{i}, \mathcal{H}\right)$ is true.

For example, in Figure 2, there are four topics and each topic has several related tweets. For topic sets $\{$ \#healthcare, \#tech, \#DataViz\}, \#healthcare.2, \#tech.2, and \#DataViz.2 are supporting tweets since they can form a size-3 clique.

Definition[Participation Ratio] The participation ratio of topic $h$ in the topic set $\mathcal{H}$ in the time-region frame $\langle w, r\rangle$ is defined as:

$$
p r_{\langle w, r\rangle}(h, \mathcal{H})=\frac{\left|\left\{v_{i} \mid s\left(v_{i}, h, \mathcal{H}\right) \wedge v_{i} \in V\langle w, r\rangle\right\}\right|}{\left|V_{\langle w, r\rangle}(h)\right|}
$$

Definition[Participation Index] The participation index 
of topic set $\mathcal{H}$ in time-region frame $\langle w, r\rangle$ is defined as:

$$
p i_{\langle w, r\rangle}(\mathcal{H})=\min _{h \in \mathcal{H}} p r_{\langle w, r\rangle}(h, \mathcal{H})
$$

For example, in Figure 2, topic \#healthcare has 4 related tweets in the given time-region frame and topic \#tech has 3 . The tweets of them form 3 neighbor relations. Assuming $\mathcal{H}=\{\#$ healthcare, \#tech $\}, \operatorname{pr}(\#$ healthcare, $\mathcal{H})=$ $3 / 4=0.75$ and $\operatorname{pr}(\#$ tech, $\mathcal{H})=3 / 3=1$. So $p i(\mathcal{H})=$ $\min \{0.75,1\}=0.75$.

Definition[Topic Association] A topic set $\mathcal{H}$ is a topic association if its participation index meets a given threshold, and such topic set will be denoted as $\mathcal{H}_{c}$.

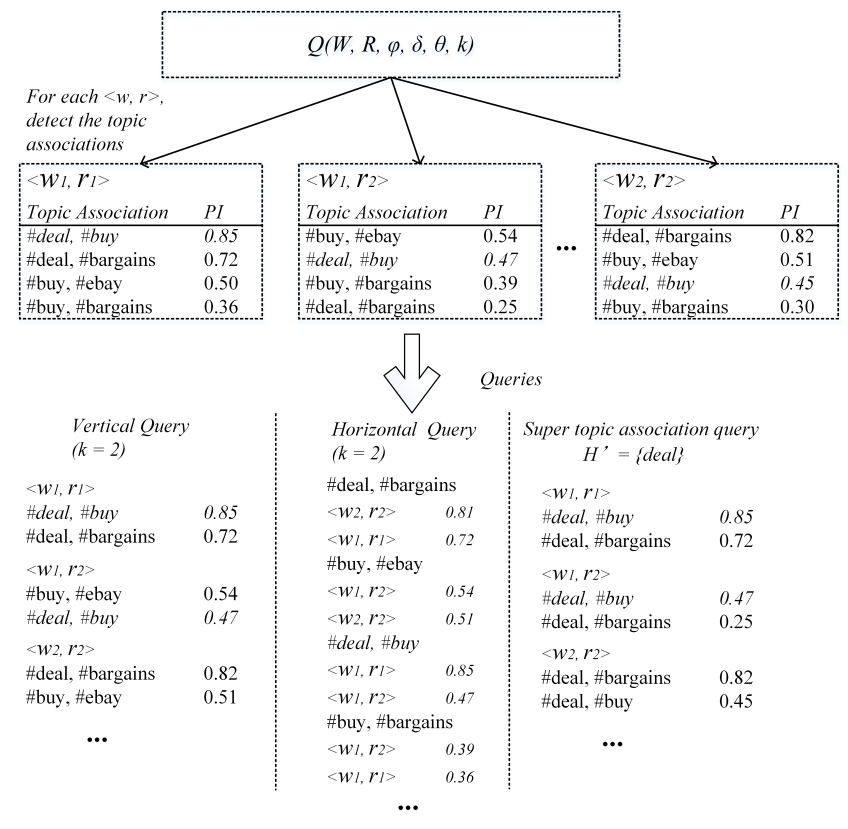

Figure 3: Vertical query, horizontal query, and super topic association query

\subsection{Query}

Each query $q\left(W_{q}, R_{q}, \varphi_{q}, \delta_{q}, \theta, k\right)$ contains six constraints: 1) A set of time windows $W_{q}=\left\{w_{1}, w_{2}, \ldots\right\}$; 2) A set of region windows $R_{q}=\left\{r_{1}, r_{2}, \ldots\right\}$; 3) Time threshold $\varphi_{q}$; 4) Distance threshold $\delta_{q}$ and 5) A user given participation index threshold $\theta ; 6$ ) a rank threshold $k$. Based on the return of queries, we define two kinds of queries: vertical query and horizontal query. Then we replace $k$ with a topic set to define super topic association query which allows a user to find other topics associated with a topic set that he is interested in.

\subsubsection{Vertical Query}

A vertical query $q_{v}\left(W_{q}, R_{q}, \varphi_{q}, \delta_{q}, \theta, k\right)$ includes five constraints and a ranking threshold $k$. The Cartesian product of $W_{q}$ and $R_{q}$ defines a set of time-region frames, which are all the possible combinations of time and region windows, $\left\{\langle w, r\rangle \mid w \in W_{q} \wedge r \in R_{q}\right\}$. Each frame corresponds to a set of tweets $V_{\langle w, r\rangle}$. For each time-region frame $\langle w, r\rangle$, we will calculate the participation index of topic sets $\mathcal{H}$ using related tweets in $V\langle w, r\rangle$, and query $q_{v}$ will return the sets of topic associations with the participation indexes larger than $\theta$ and ranking in the top- $k$ among all the topic sets.

\subsubsection{Horizontal Query}

Similarly, the horizontal query $q_{h}\left(W_{q}, R_{q}, \varphi_{q}, \delta_{q}, \theta, k\right)$ also has a ranking threshold $k$. For each topic set $\mathcal{H}$, we calculate its participation indexes $p i_{\langle w, r\rangle}(\mathcal{H})$ in different time-region frames $\langle w, r\rangle$ and then return the time-region frames with top- $k$ highest values of $p i_{\langle w, r\rangle}(\mathcal{H})$. The number of returned frames may be less than $k$ if there are less than $\mathrm{k}$ frames having participation index higher than the given threshold $\theta$.

\subsubsection{Super Topic Association Query}

The super topic association query, $q_{h}\left(W_{q}, R_{q}, \varphi_{q}, \delta_{q}, \theta, \mathcal{H}^{\prime}\right)$, replaces $k$ with a set of topics/hashtags $\mathcal{H}^{\prime}$. For each timeregion frames $\langle w, r\rangle$ in $W_{q} \times R_{q}$, it returns topic associations $\mathcal{H}$ where $\mathcal{H}^{\prime} \subsetneq \mathcal{H}$ and $p i_{\langle w, r\rangle}(\mathcal{H})$ larger than $\theta$.

For example, Figure 3 shows the results of vertical query and horizontal query. $W$ contains two time windows $w_{1}$ and $w_{2}$, and $R$ contains two regions $r_{1}$ and $r_{2}$. Assuming $\theta=0.25$, there are several hashtags and they can form topic associations in different time-regions frames. The participation index of each topic association is shown in the figure. For vertical query, assuming $k=2$, it will return the top-2 topic associations in each frame. For example in $\left\langle w_{1}, r_{1}\right\rangle$, it returns $\{\#$ deal, \#buy\} and $\{\#$ deal, \#bargains $\}$ since their participation indexes are larger than the threshold and ranked in the top-2. For the horizontal query, it returns the top related time-region frames. For instance, the topic association $\{\#$ deal, \#bargians\} achieves highest participation index in $\left\langle w_{2}, r_{2}\right\rangle$ and $\left\langle w_{1}, r_{1}\right\rangle$. So when $k=2$, it will return these two time-region frames for this topic association. For the super topic association query, assuming that the set of hashtags inputed is $\{\#$ deal $\}$, it will return the associations in different time-region frames which contain \#deal.

\section{MINING TOPIC ASSOCIATION}

Given the definition of the participation index, we now propose algorithms for the queries of mining topic association.

\subsection{Calculation of the Participation Index}

When considering the neighbor relationship between tweet$\mathrm{s} v_{i}$ and $v_{j}$, we redefine it as $\left|t_{i}-t_{j}\right| \leq \varphi_{q}$ and $v_{i}$ and $v_{j}$ are published in the same city because real world events are greatly affected by the geographic boundary. People may be interested in completely different topics in different cities even the distance between the two cities is short. Another reason is that sometimes we do not have an accurate location of tweet. We may only have the city level location from the tweets or from the profiles of authors. Finally, users may have a city level active area and they may publish tweets anywhere in the city they live.

From the definition of the participation ratio and index, we can see the most important step in detecting topic association is counting the number of supporting tweets of each topic $h$ in $\mathcal{H}$ and calculating the participation index. The Algorithm 1 gives the process of this step.

Previous work has developed R-tree based [26] or grid based [23] index for spatial related search engines. In this problem, the tweets $V_{\langle w, r\rangle}(h)$ of each topic $h$ are distributed in a three dimensions space, latitude, longitude, and time. Based on our definition of neighbor relationship, we use the bitmap index as the Topic Geographic Index $\mathcal{I}_{T}$ as shown in 


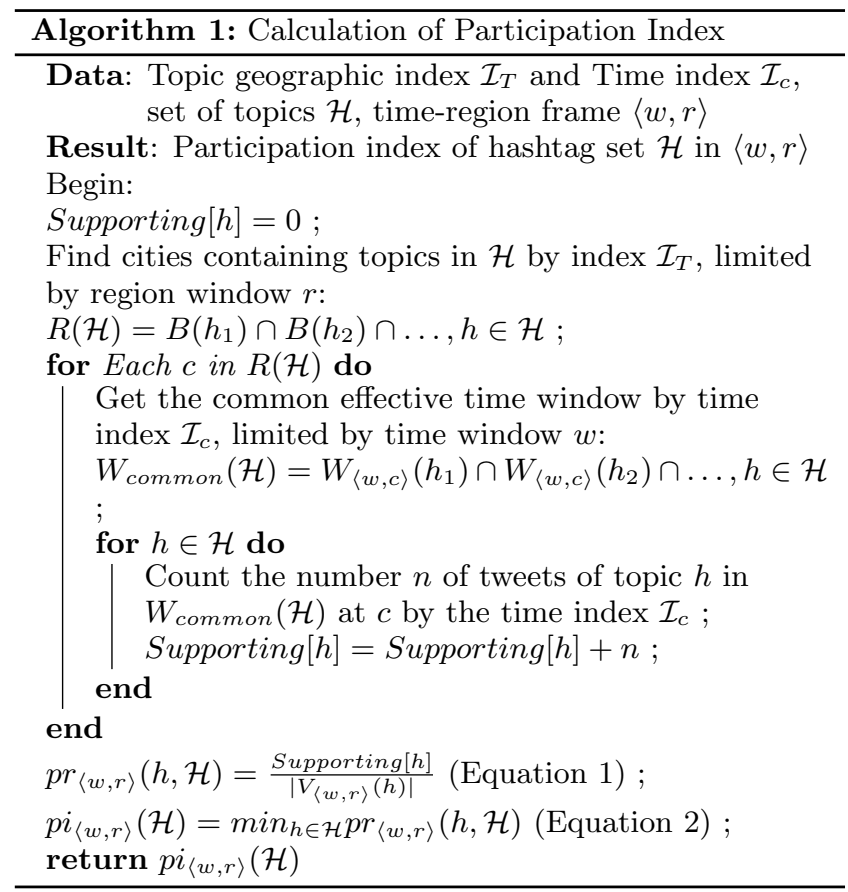

Figure 4. For each topic $h, \mathcal{I}_{T}$ contains the index $\mathcal{I}_{T}(h)$ of $h$ which records the geographic distribution of tweets among cities related to topic $h$. If we divide the region window $r$ into city level as: $r=\left\{c_{1}, c_{2}, \ldots\right\}$, where $c$ represents a city level region window, we can denote $V_{\langle w, r\rangle}(h)$ as $\left\{V_{\left\langle w, c_{1}\right\rangle}(h) \cup\right.$ $\left.V_{\left\langle w, c_{2}\right\rangle}(h) \cup \ldots\right\}$. Each time index in a city $c$ corresponds to a set of tweets $V_{\langle w, c\rangle}(h)$ if it is not empty (topic $h$ has at least one tweet in region $c$ during the time windows in $w$ ), and we denote the set of these cities $c$ as $B(h)$.

Then we apply an Time index $\mathcal{I}_{c}(h)$ on the time dimension on each set of tweets $V_{\langle w, c\rangle}(h)$ as it is shown in Figure 4. Every tweet $v_{i}$ of topic $h$ at city $c$ has an "effective period" $\left[t_{i}-\varphi_{q}, t_{i}+\varphi_{q}\right]$ defined by the user given time threshold $\varphi_{q}$ in the query. The union of the effective periods of tweets in $V_{\langle w, c\rangle}(h)$ is the total effective periods of topic $h$ in the city $c$ and denoted as $W_{\langle w, c\rangle}(h)$. So based on the definition of neighbor relationship, for a tweet $v_{j}$ of another topic, if $v_{j}$ was published at city $c$ and during the period of $W_{\left\langle W_{q}, c\right\rangle}(h)$, we can find at least one tweet $v_{i}$ of topic $h$ which has neighbor relation with $v_{j}$.

Algorithm 1 provides the process of the calculation of participation index. Given a set of topics $\mathcal{H}$ and the time-region frame $\langle w, r\rangle$, we firstly find the cities which contain tweets of every topic $h$ in $\mathcal{H}$. For each topic $h$, we have the topic geographic index $\mathcal{I}_{T}(h)$ to help to get the set of cities which have related tweets of $h$ and we denote the set of cities as $B(h)$. So the intersection of all $B(h), h \in \mathcal{H}$, is the set $R(\mathcal{H})$ of cities containing related tweets of every topic in $\mathcal{H}$.

Based on our definition of neighbor relation, for a tweet $v_{i}\left(t_{i}, l_{i},\left\{h_{i}\right\}\right), l_{i} \in c$, we can find a neighbor $v_{j}$ related to another topic topic $h_{j}$ only when $h_{j}$ has tweets published in city $c$ and $t_{i} \in W_{\langle w, c\rangle}\left(h_{j}\right)$. For a set of topics, we can calculate the effective period $W_{\langle w, c\rangle}(h)$ of each topic in a city $c$. The common effective period of the topics is denoted as $W_{\text {common }}(\mathcal{H})$. For each topic $h$ in $\mathcal{H}$, if it has a related tweet $v$ published in $W_{\text {common }}(\mathcal{H})$ in city $c$, we can find

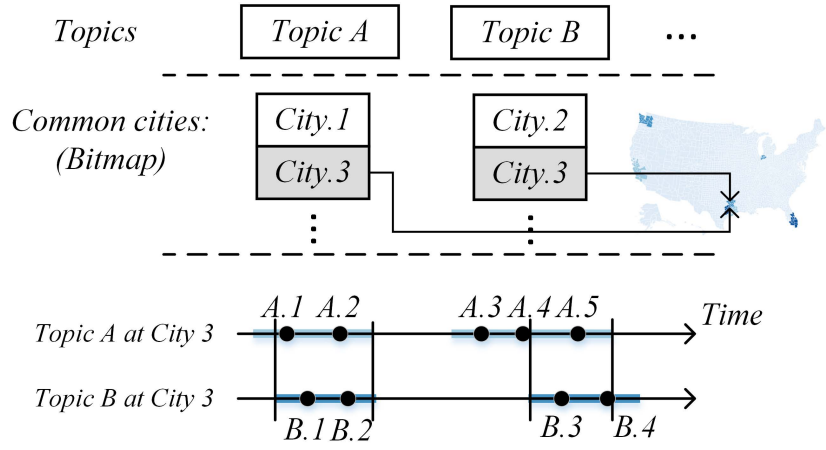

Figure 4: Calculation of participation index of topic sets $\mathcal{H}$ : There are two topics in this example, \#Topic $A$ and $B$. \#Topic $A$ has related tweets distributed in two cities. We use the bitmap index $\mathcal{I}_{T}$ to record cities which has tweets related to \#Topic A. For the tweets in each city, e.g. the tweets related to \#Topic A in City.3, we will use the Time Index $\mathcal{I}_{c}$ to record the beginning and ending time of the effective period and the tweets published during these periods.

at least one neighbor tweet from other topics in $\mathcal{H}$. So $v$ will be a supporting tweet for $\mathcal{H}$. In Algorithm 1, for each city $c$ in $R(\mathcal{H})$, we use the time index of tweets in city $c$ of topic $h, \mathcal{I}_{c}(h)$, which records the begin and end time of each effective period and the tweets published during the period, to achieve the common period $W_{\text {common }}(\mathcal{H})$. Tweets of each topic in $W_{\text {common }}(\mathcal{H})$ will be counted as supporting tweet for $\mathcal{H}$. After scanning all the cities in $R(\mathcal{H})$, we can calculate the participation ratio of $h$ by the number of supporting tweets and get the participation index of $\mathcal{H}$.

\subsection{Calculation of Horizontal Query}

For the horizontal query, we need to generate all the topic associations in each time-region frame. Algorithm 2 provides this process. For each $\langle w, r\rangle$ frame in $W_{q} \times R_{q}$, we firstly put every topic in the set of a size-1 topic association $\left\{\mathcal{H}_{c}\right\}_{1}^{\langle w, r\rangle}$. In each loop, we use the apriori-gen algorithm [2] to generate size- $n$ topic association candidates $\{\mathcal{H}\}_{n}$ by $\operatorname{size}-(n-1)$ topic association $\left\{\mathcal{H}_{c}\right\}_{n-1}^{\langle w, r\rangle}$. Then we use the Algorithm 1 to calculate the participation index of every candidates and get the size- $n$ topic association. After generating topic associations in every $\langle w, r\rangle$ frames, we will return every topic association $\mathcal{H} \in\left\{\mathcal{H}_{c}\right\}$ and the top-k frames which $\mathcal{H}$ achieves the highest participation index.

\subsection{Calculation of Vertical Query}

As shown in Algorithm 3, for each time-region frame $\langle w, r\rangle$, after we generate size-n topic association, we will prune the topic association by removing the association ranking out of $k$ in $\left\{\mathcal{H}_{c}\right\}^{\langle w, r\rangle}$ because such topic association cannot be a subset of a topic association which has a higher participation index.

\subsection{Calculation of Super Topic Association Query}

The algorithm of super topic association query is based on the method of vertical query. The difference is that the process will begin at generating size- $\left(\left|\mathcal{H}^{\prime}\right|+1\right)$ topic association candidates and these candidates must contain all the hashtags in $\mathcal{H}^{\prime}$. 

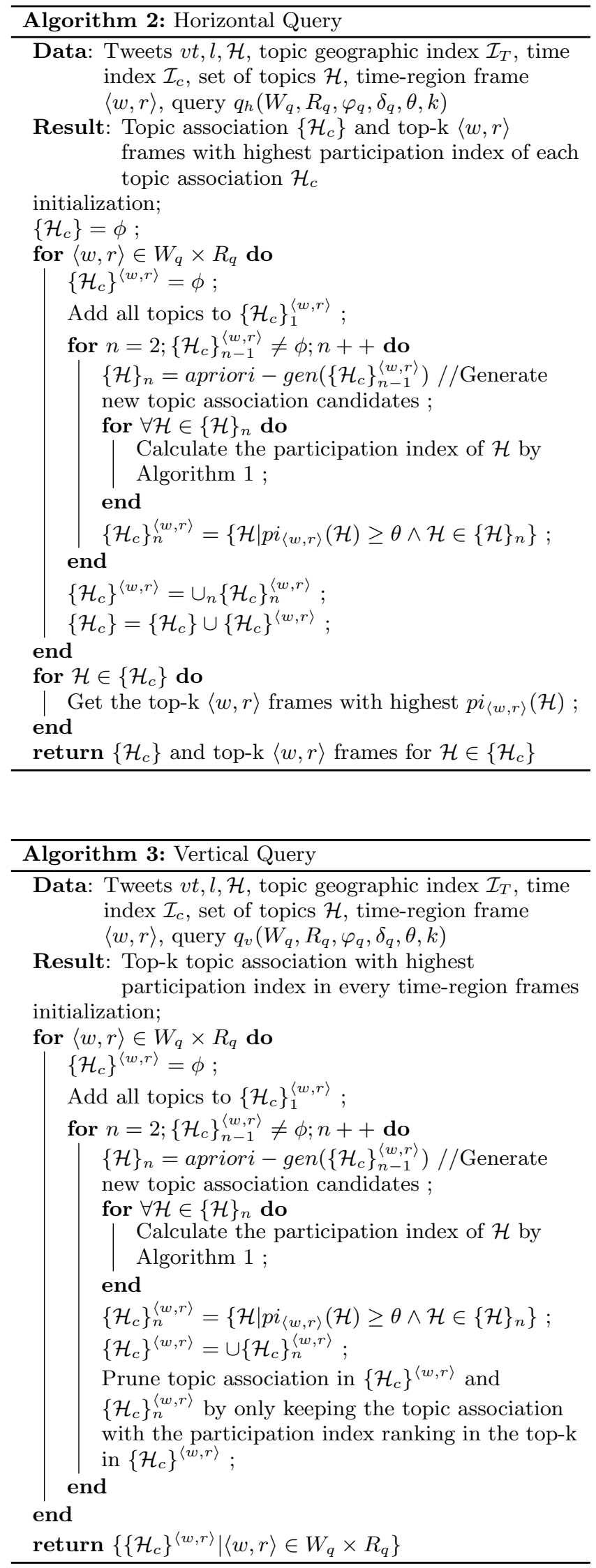

\section{OPTIMIZATION OF QUERYING RESULT}

\subsection{Topic Combination}

Many topic pairs or groups refer to the same thing or same event. These topics may share similar words and be talked in same area and same time (e.g. \#amazonwishlist and \#amazoncart). Some official Twitter accounts even list several hashtags and tell users that all these hashtags are related to the same thing, for example, \#choicetvchemistry and \#choicetvliplock in Table 2. If such a hashtag group contains $n$ hashtags, it may generate $2^{n}$ topic associations. It increases computation time and leads to a huge number of redundant associations. So before we apply our algorithm, we will combine some hashtags which are related to the same thing together to make the algorithm more efficient, and less number of detected results can help to extract useful topic associations more easily.

We combine the hashtags in two steps: First, we build a hashtag similarity graph. Each hashtag is a node in this graph and there is an edge between every hashtag pair. We assign each edge a weight as:

$$
\begin{aligned}
& \text { Weight( } \left.\text { hashtag }_{1}, \text { hashtag }_{2}\right) \\
& =\frac{\mid \text { Total longest common substring }\left.\right|_{\text {max }\left\{\mid \text { hashtag }_{1}|,| \text { hashtag }_{2} \mid\right\}}}{\text { mash }}
\end{aligned}
$$

We follow the method in [7] to get the longest substring. We remove the longest common substring of the two hashtags iteratively until there is no common substring longer than 1. For example, the longest substring of \#amazonwishlist and \#amazoncart is "amazon" and the weight of the edge is 0.43 .

Then we run the hierarchical clustering algorithm on this graph. The distance between two clusters is defined as the average weight of edges between hashtags in these two clusters. However, we have two other problems: 1) when we stop the hierarchical clustering, some hashtags share long common substrings but actually they do not refer to the same thing (e.g. \#braves and \#travel). Table 2 lists more examples. All these examples are similar strings but their participation index is 0 .

To solve these problems, we introduce the participation index here. Before we combine two clusters together, we will calculate the participation index of the new cluster. If it is less than the threshold, we won't combine them and we will go to next two clusters with highest average weight of edges. If all the top- $\mathrm{N}$ candidates cannot meet the requirement, we will stop the hierarchical clustering. Table 2 shows that the use of participation index in topic clustering can filter out hashtags "similar in appearance but dissimilar in spirit".

\subsection{Topic Filtering}

Many topics follows a rhythm of a typical day. For example, they happen more when there are more people using Twitter such as lunch time. These topics such as job advertisement are white noises and do not related to other topics. Here we propose a method to filter out these topics to prevent them from participating in most topics associations.

In a time-region frame $\langle w, r\rangle$, we calculate the correlation coefficient between topic $h$ and all the topics by their distributions of number of tweets in each city $c$ in $r$ during the time window $w$ as: 
Table 2: Apply participation index in topic combination

\begin{tabular}{|c|c|c|}
\hline Topic Pairs & Wt & PI \\
\hline \hline tamuc, tamuk & 0.8 & 0 \\
\hline longlivela, longlivejr & 0.8 & 0 \\
\hline rays, raya & 0.75 & 0 \\
\hline live, livy & 0.75 & 0 \\
\hline \hline freedom, freedex & 0.71 & 0 \\
\hline amazoncart, amazonwishlist & 0.42 & 0.95 \\
\hline phillies, philly & 0.625 & 0.81 \\
\hline nationaldonutday, nationaldoughnutday & 0.375 & 0.68 \\
\hline choicetvchemistry, choicetvliplock & 0.47 & 0.48 \\
\hline
\end{tabular}

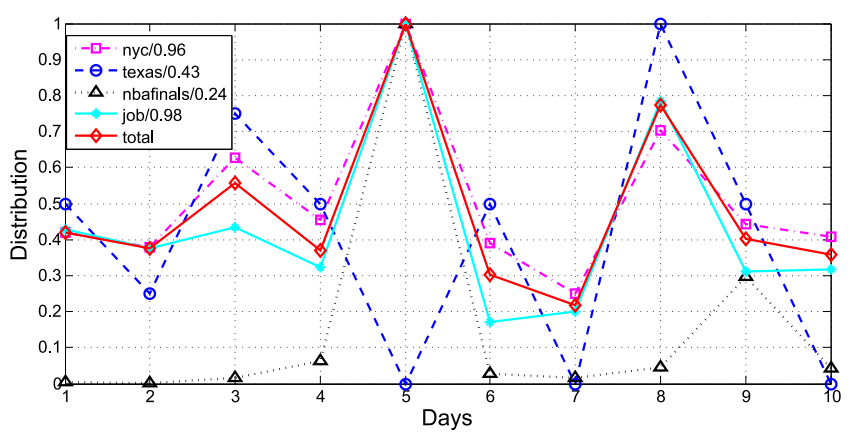

(a) New York

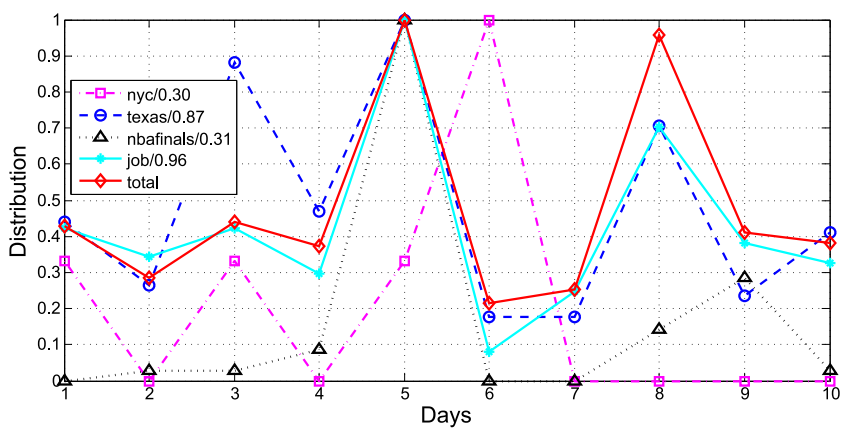

(b) Austin

Figure 5: The distribution of tweets of different topics in New York and Austin.

$\operatorname{Cor}\left(h, \mathcal{H}_{\text {total }}\right)=\frac{\sum_{u=1}^{n}\left(\left|v_{u}^{h}\right|-\overline{\left|v_{u}^{h}\right|}\right)\left(\left|v_{u}^{\mathcal{H}}\right|-\overline{\left|v_{u}^{\mathcal{H}}\right|}\right)}{\sqrt{\sum_{u=1}^{n}\left(\left|v_{u}^{h}\right|-\overline{\left|v_{u}^{h}\right|}\right)^{2} \sum_{u=1}^{n}\left(\left|v_{u}^{\mathcal{H}}\right|-\overline{\left|v_{u}^{\mathcal{H}}\right|}\right)^{2}}}$

Here, $V_{\langle u, c\rangle}(h)=\left\{v_{1}^{h}, v_{2}^{h}, \ldots\right\}, V_{\langle u, c\rangle}(\mathcal{H})=\left\{v_{1}^{\mathcal{H}}, v_{2}^{\mathcal{H}}, \ldots\right\}$, and $u$ denotes a unit of time, such as one day.

When a topic correlates with total volume of the tweets above a threshold, there tweets are considered as uninteresting and are excluded from the topic association mining. Figures 5(a) shows that \#nyc is a topic highly correlated to the number of tweets in New York during a day while \#texas (correlation index of 0.87 ) is not. In Austin however as shown in Figure 5(b), \#texas is a highly correlated topic (correlation index of 0.87 ) while \#nyc and \#nbafinals are not. \#job is a highly correlated topic in both cities. So the topics like \#job will not be considered in topic association mining since such topics are not effected by other topics or real world events.

\section{EXPERIMENT}

In this section, we first introduce the data set used in the experiment. Then we evaluate the effect of parameters of queries by comparing the running time of each query. Finally, we demonstrate the experiment results in some timeregion frames to explain the meaning of the topic association. We show the results of topic filtering. We compare the results produced by our algorithms with human judges.

\subsection{Data}

We collected tweets published from May 21 to June 15, 2015 using Twitter Streaming API $^{1}$ with the spatial bounding box $\left[24^{\circ} 3^{\prime} N, 127^{\circ} 7^{\prime} \mathrm{W}, 48^{\circ} 8^{\prime} N, 65^{\circ} 5^{\prime} \mathrm{W}\right]$, which covers U.S. mainland. The dataset consists $27,956,257$ tweet$\mathrm{s}$ from 492,492 topics. We removed the topics with less than 1,000 related tweets, and finally we selected 10,201,960 tweets which contain both geotag and hashtag from 2,000 topics.

\subsection{Detecting Topic Association}

Firstly, we evaluate the effect of different parameters on the running time. Table 3 gives the list of parameters and the default values.

\section{Number of Topics}

Figure 6(a) shows the running time of vertical query and horizontal query under different number of topics. More topics means more topic associations under the same settings and the increase of number of topic associations can be exponential in the worst case. The running time in Figure 6(a) shows this trend. In our algorithm, each topic will have a time index in each city and a geographic index to record its distribution. So the increase of the number of topics will take more memory to store the indexes but it has little influence on the running time of calculating the participation index.

\section{Time-region Frame}

In this experiment, we use four parameters to describe the time-region frame. $W_{q}$ and $R_{q}$ are largest time and region frame and $W_{\text {gran }}$ and $R_{\text {gran }}$ are use used to divide the largest time and region frames into smaller ones, i.e. we divide $W_{q}$ and $R_{q}$ based on the length of $W_{g r a n}$ and $R_{\text {gran }}$.

Firstly, we test the influence of the region window $R_{q}$. We divide the cities in U.S. into 1 to 32 groups equally and record the average querying time on these groups. Figure 6 (b) gives the results of this experiment. The numbers on x-axis represent the $R_{\text {gran }}$ proportion of each group of the total region window. For example $1 / 2$ means that we divide the whole area of the U.S. into two groups and y-axis is the average running time of the queries on these two groups. The results show that the running time has a linear relationship with the scope of the region window, e.g. the running time of two groups is about half of the "total" case and twice as much as the " $1 / 4$ " case. The two queries perform differently in the "city" case, where we set each city as the region window. Running the queries in city granularity will generate a large number of topic associations. So the horizontal query has

\footnotetext{
${ }^{1}$ https://dev.twitter.com/streaming/overview
} 
Table 3: Parameters used in queries

\begin{tabular}{|c||c|c|c|}
\hline Parameter & Definition & Default value & Test scope \\
\hline \hline$N_{\mathcal{H}}$ & The total number of topics used & 1,000 & {$[100,2000]$} \\
\hline$W_{q}$ & The total time windows & 20 days & {$[1,20]$} \\
\hline$R_{q}$ & The total region window & US & {$[$ city level, U.S.] } \\
\hline$W_{\text {gran }}$ & The granularity of time window & 1 & {$[1,20]$} \\
\hline$R_{\text {gran }}$ & The granularity of region window & 1 & {$[$ city level, U.S.] } \\
\hline$\varphi_{q}$ & Time threshold in the definition of neighbor relation & fixed \\
\hline$\delta_{q}$ & Distance threshold in the definition of neighbor relation & City level & fixed \\
\hline$\theta$ & The threshold of the participation index & 0.8 & {$[0.040 .8]$} \\
\hline$k$ & The ranking threshold of the query & 40,000 & {$[50040,000]$} \\
\hline
\end{tabular}

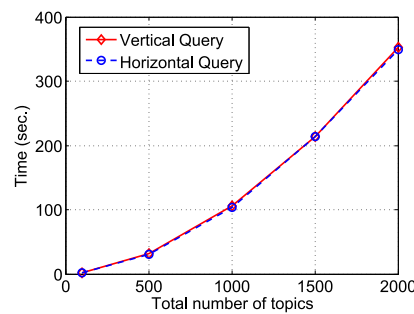

(a) Number of topics $N_{\mathcal{H}}$

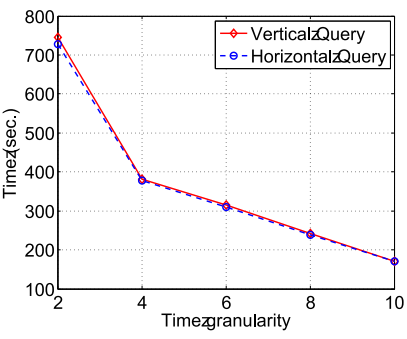

(e) $W_{\text {gran }}$

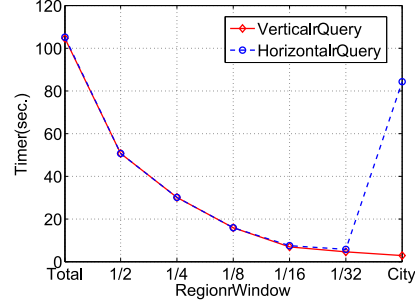

(b) Region window $R_{q}$

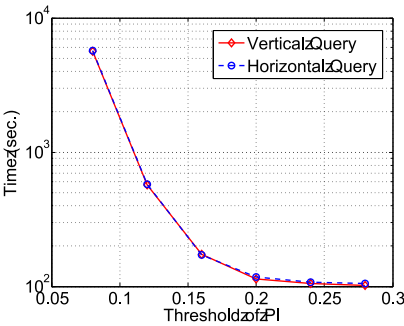

(f) $\theta$

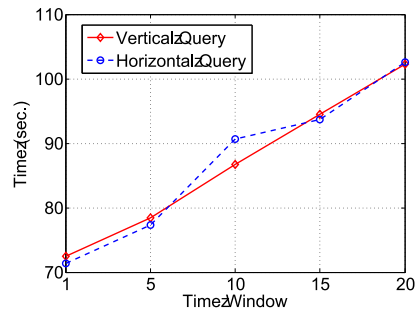

(c) Time window $W_{q}$

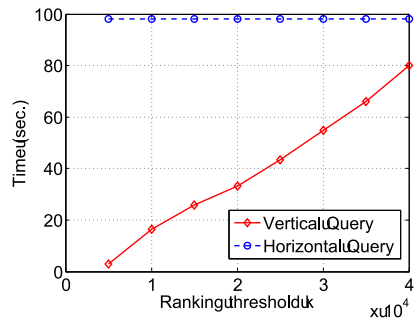

(g) Ranking threshold $k$ (1)

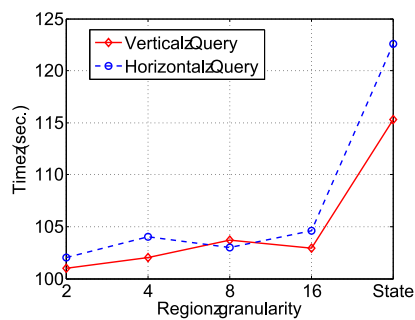

(d) $R_{\text {gran }}$

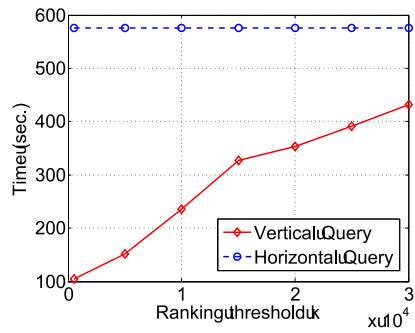

(h) Ranking threshold $k(2)$

Figure 6: Running time under different setting of parameters.

a longer running time since vertical query can prune more topic association candidates. We will provide more analysis in the following section.

The influence of time window size is shown in Figure 6(c). Similar as the results of changing the total region window, the running time has linear relationship with the time window. The increase of time and region window needs additional indexes to record the distribution of new tweets. If we only increase the region window, for each topic set, we need to scan more cities to calculate the participation. If we increase the time window only, for each city we need to scan a larger time window by the time index. That explains why the running time increases linearly with the region and time window.

Then we fixed the largest time and region windows and change the granularity of the sub-time and sub-region windows. If we set smaller smaller time or region window, each query will be faster, but the query needs to be executed more times. When changing the granularity of the region window (Figure 6(d)), we can see that the total running time does not change too much. It only increases in the "state" case because some state only contains one city and the query will take longer time. Figure 6(e) is the results of the running time of different time window granularities. The total running time can be smaller when we set a longer time window granularity. Actually, we can estimate the results in Figure 6(d) and 6(e) by the results in Figure 6(b) and 6(c).

\section{Threshold on Participation Index}

The threshold of the participation index greatly affects the number of topic associations. As shown in Figure 6(f), with the decreasing of the value of the threshold $\theta$, the running time increase quickly since there will be an exponential growth of the number of topic associations. Here the ranking threshold $k$ of the vertical query is big enough, so the optimization is not useful and the running time of both the two queries is similar.

\section{Top-K Selection}

In Figure 6(g) and 6(h), we test the influence of the ranking threshold $k$ of vertical query. In these two experiments, we set the value of the threshold of participation index $\theta$ as 0.8 and 0.12 separately. In both the two cases, the ranking threshold greatly affects the running time. So when this threshold is not large enough, during the generating step, we can prune a part of topic associations which have their participation index larger than the threshold $\theta$. So the running time of the vertical query will be much smaller than the horizontal query. With the increase of the ranking threshold $k$, the gap of the running time of the two queries becomes smaller.

\subsection{The Topic Association}

In this experiment, we use the results of the two queries 
Table 4: Vertical Query

\begin{tabular}{|c||c|c|c|}
\hline City: & New York & Chicago & Houston \\
\hline \hline 1 & braves, bruins & rockets, rays & traffic, houstonastros \\
\hline 2 & nationaldonutday, uclfinal & astros, rockets & traffic, houstontexans, houstonastros \\
\hline 3 & sundayfunday, sunday & astros, rockets, rays & traffic, houstontexans \\
\hline 4 & gsw, nbafinals2015 & astros, rays, texasedmfamily & houstontexans, houstonastros \\
\hline 5 & nbafinals, cle & rockets, rays, texasedmfamily & whodatnation, gramfam \\
\hline
\end{tabular}

to show that our algorithm can detect meaningful topic associations. We also compare the results with/without the topic filtering method.

\section{Vertical Query}

Table 4 shows the top- 5 topic associations (sorted by participation index) in New York, Chicago, and Houston from May 25 to Jun 15. The relationship between hashtags in most topic associations can be easily revealed by investigating the real world event they refer to. For example, "\#gsw, \#nbafinals2015" and "\#nbafinals, \#cle", \#gsw and \#cle refer to the two teams which play against in the NBA final, 2015.

\section{Horizontal Query}

Table 5: Horizontal Query

\begin{tabular}{|c||c|c|}
\hline & gsw, nbafinals2015 & nbafinals, cle \\
\hline \hline Frame 1 & New York, June 7 & New York, June 7 \\
\hline Frame 2 & New York, June 4 & New York, June 4 \\
\hline Frame 3 & New York, June 16 & New York, June 14 \\
\hline
\end{tabular}

Table 5 shows examples of horizontal query results. Here we select two topic associations in Table 4, gsw, nba finals 2015 and nbafinals, cle. For the first topic association, the days when it achieve highest participation indexes is June 7, 4, and 16 , and for the second example, they are June 7,4 , and 14. If we check the schedule of NBA finals in 2015, we will find that there exists a match in all these days. So we can see that the horizontal query will return the most related time-region frames.

\section{Super Topic Association Query}

Table 6 lists two examples for super topic association query. We set $\mathcal{H}^{\prime}$ as $\{\#$ nbafinals $\}$ and $\{\#$ amazoncart $\}$ and the query returns the topic associations containing those hashtags.

Table 6: Super Topic Association Query

\begin{tabular}{|c|c||c|c|}
\hline \#nbafinals & $P I$ & \#amazoncart & $P I$ \\
\hline \hline \#gsw & 0.79 & \#amazonwishlist & 0.95 \\
\hline \#cle & 0.76 & \#einkaufen & 0.94 \\
\hline \#nbafinals 2015 & 0.71 & \#bargains & 0.92 \\
\hline
\end{tabular}

\section{Topic Filtering}

In Table 7 we compare the querying results with and without the topic filtering. We apply the same settings with the experiment for vertical query. Here we list the top-5 topic associations which have the highest participation indexes in
New York from May 25 to Jun 15. If we do not apply the topic filtering method, we will detect lots of topic associations including nyc, newyork and job,hiring. They have a very high participation index because people discuss about them every often and they can form supporting cliques easily. After we filter such topics, we can see that Wthe new results are more meaningful. Most of them are caused by some real world event.

Table 7: Topic Filtering

\begin{tabular}{|c|c|}
\hline Before Filtering & After Filtering \\
\hline \hline nyc, newyork & braves, bruins \\
\hline job, hiring & nationaldonutday, uclfinal \\
\hline job, nyc & sundayfunday, sunday \\
\hline job, newyork & gsw, nbafinals2015 \\
\hline hiring, jobs & nbafinals, cle \\
\hline
\end{tabular}

\subsection{Effectiveness of Participation Index}

To verify the effectiveness of participation index, which greatly effects the final results, we compare the experimen$\mathrm{t}$ results with human judgment in this experiment. Since it is difficult to give an appropriate measurement to judge the tightness between topics, we only compare the tightness of topic sets. First we create topic set groups. Each topic set group contains 5 topic sets and each topic sets contain two topics. The participation indexes of these five topic sets should be in the range of $(0,0.05],(0.05,0.15]$, $(0.15,0.3],(0.3,0.45]$, and $(0.45,1]$ respectively. The gap of participation indexes of each two topic set should be larger than 0.05. Based on the participation indexes, we can get a rank of these topic sets $X$. Then we manually compare the tightness of topic sets in the same group and give a rank $Y$ based on their judgment. To make the judgment easier and more accurate, the topic sets in the same group will share a common topic. Finally, we use Spearman's rank correlation coefficient to compare the human judgment with the experiment (rank based on the participation index).

$$
\rho=1-\frac{6 \sum\left(x_{i}-y_{i}\right)^{2}}{n\left(n^{2}-1\right)}
$$

The $x_{i}$ and $y_{i}$ is the ranking of the $i$ th element in the topic set group given by participation index and human judgment. Since we fix the size of topic set group as five, the value of $n$ is 5. The average value of the Spearman's rank correlation of our 200 cases is 0.68 , which illustrates that the participation index can measure the tightness of topics effectively. Figure 7 shows the distribution of the values of the Spearman's rank correlation coefficient. Most cases have the Spearman's rank correlation coefficient larger than 0.4 and 40 percent cases have the value larger than 0.8 . 


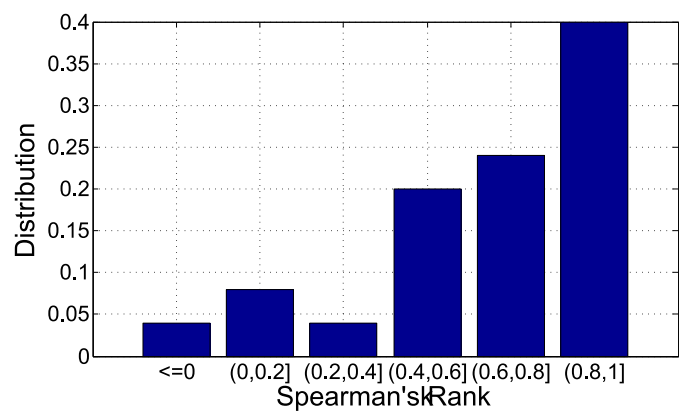

Figure 7: Compare the ranking based on the participation indexes with the human judgment.

\section{CONCLUSION}

In this paper, we apply participation index as the measurement of topic closeness. We define three different kinds of queries to mining the topic association. For each query, we design the algorithm for it. Two optimization methods, topic filtering and topic combination are used to help getting a better query results. We test our method on a large Twitter data set with 27,956,257 tweets which contain both hashtags and geo-tag. The results of the experiment show that the mining algorithms are effective and efficient. We also compare the experimental results with human judgment to verify the effectiveness of using participation index to measure the closeness among topics.

\section{REFERENCES}

[1] H. Achrekar, A. Gandhe, R. Lazarus, S.-H. Yu, and B. Liu. Predicting flu trends using twitter data. In INFOCOM WKSHPS, pages 702-707. IEEE, 2011.

[2] R. Agrawal, R. Srikant, et al. Fast algorithms for mining association rules. In $V L D B$, volume 1215 , pages 487-499, 1994.

[3] S. Asur and B. A. Huberman. Predicting the future with social media. In WI-IAT, volume 1 , pages 492-499. IEEE, 2010.

[4] C. Budak, T. Georgiou, D. Agrawal, and A. El Abbadi. Geoscope: Online detection of geo-correlated information trends in social networks. VLDB, 7(4):229-240, 2013.

[5] C. Budak, T. Georgiou, and D. A. A. El Abbadi. Geowatch: Online detection of geo-correlated information trends in social networks. Technical report, Technical report, UCSB, 2013.

[6] S. Carter, M. Tsagkias, and W. Weerkamp. Twitter hashtags: Joint translation and clustering. ACM WebSci, 2011.

[7] C. Friedman and R. Sideli. Tolerating spelling errors during patient validation. Computers and Biomedical Research, 25(5):486-509, 1992.

[8] K. Glasgow and C. Fink. Hashtag lifespan and social networks during the london riots. In Social Computing, Behavioral-Cultural Modeling and Prediction, pages 311-320. Springer, 2013.

[9] Y. Huang, S. Shekhar, and H. Xiong. Discovering colocation patterns from spatial data sets: a general approach. TKDE, 16(12):1472-1485, 2004.
[10] K. Y. Kamath, J. Caverlee, K. Lee, and Z. Cheng. Spatio-temporal dynamics of online memes: a study of geo-tagged tweets. In $W W W$, pages $667-678$. International World Wide Web Conferences Steering Committee, 2013.

[11] S. M. Kywe, T.-A. Hoang, E.-P. Lim, and F. Zhu. On recommending hashtags in twitter networks. In Social Informatics, pages 337-350. Springer, 2012.

[12] R. Lee, S. Wakamiya, and K. Sumiya. Discovery of unusual regional social activities using geo-tagged microblogs. $W W W, 14(4): 321-349,2011$.

[13] A. M. MacEachren, A. C. Robinson, A. Jaiswal, S. Pezanowski, A. Savelyev, J. Blanford, and P. Mitra. Geo-twitter analytics: Applications in crisis management. In 25th International Cartographic Conference, pages 3-8, 2011.

[14] R. Munro, S. Chawla, and P. Sun. Complex spatial relationships. In ICDM, pages 227-234. IEEE, 2003.

[15] S. Petrović, M. Osborne, and V. Lavrenko. Streaming first story detection with application to twitter. In NAACL, pages 181-189. Association for Computational Linguistics, 2010.

[16] T. Sakaki, M. Okazaki, and Y. Matsuo. Earthquake shakes twitter users: real-time event detection by social sensors. In $W W W$, pages 851-860. ACM, 2010.

[17] T. Sakaki, M. Okazaki, and Y. Matsuo. Tweet analysis for real-time event detection and earthquake reporting system development. TKDE, 25(4):919-931, 2013.

[18] A. Schulz, A. Hadjakos, H. Paulheim, J. Nachtwey, and M. Mühlhäuser. A multi-indicator approach for geolocalization of tweets. In ICWSM, 2013.

[19] S. Shekhar and Y. Huang. Discovering spatial co-location patterns: A summary of results. In $S S T D$, pages 236-256. Springer, 2001.

[20] T. Sugitani, M. Shirakawa, T. Hara, and S. Nishio. Detecting local events by analyzing spatiotemporal locality of tweets. In WAINA, pages 191-196. IEEE, 2013.

[21] R. Tinati, L. Carr, W. Hall, and J. Bentwood. Identifying communicator roles in twitter. In $W W W$, pages 1161-1168. ACM, 2012.

[22] O. Tsur and A. Rappoport. What's in a hashtag?: content based prediction of the spread of ideas in microblogging communities. In WSDM, pages 643-652. ACM, 2012.

[23] S. Vaid, C. B. Jones, H. Joho, and M. Sanderson. Spatio-textual indexing for geographical search on the web. In SSTD, pages 218-235. Springer, 2005.

[24] L. Yang, T. Sun, M. Zhang, and Q. Mei. We know what@ you\# tag: does the dual role affect hashtag adoption? In $W W W$, pages 261-270. ACM, 2012.

[25] X. Zhang, N. Mamoulis, D. W. Cheung, and Y. Shou. Fast mining of spatial collocations. In $A C M S I G K D D$, pages 384-393. ACM, 2004.

[26] Y. Zhou, X. Xie, C. Wang, Y. Gong, and W.-Y. Ma. Hybrid index structures for location-based web search. In $C I K M$, pages $155-162$. ACM, 2005. 